\title{
Clinically unexpected lymph node: An old enemy to remember in the cancer diagnosis and treatment
}

\author{
İmdat Eroğlu ${ }^{1 *}$, Burak Yasin Aktaş², Olcay Kurtulan ${ }^{3}$, Deniz Can Güven² and Sercan Aksoy ${ }^{2}$ \\ ${ }^{1}$ Department of Internal Medicine, Hacettepe University Faculty of Medicine, Turkey \\ ${ }^{2}$ Department of Medical Oncology, Hacettepe University Faculty of Medicine, Turkey \\ ${ }^{3}$ Department of Pathology, Hacettepe University Faculty of Medicine, Turkey
}

\section{Introduction}

Tuberculosis is a common infection especially in the developing countries and it can be detected coincidentally on imaging done for other purposes, such as cancer detection and staging. Treatment of tuberculosis is challenging among cancer patients, due to drug interactions caused by anti-tuberculosis treatment.

\section{Objectives}

To describe a patient with tuberculosis lymphadenitis detected incidentally on PET-CT during a staging of newly diagnosed early stage breast cancer and its effect on our treatment decision.

\section{Case report}

A 53-year old female patient admitted to clinic with a mass on her right breast. Her past medical history was unremarkable. The breast ultrasound revealed a 29x11 mm solid lesion located $4 \mathrm{~cm}$ away from nipple on 7 o'clock position and there was no axillary lymphadenopathy.

A tru-cut biopsy was performed and showed grade 2 invasive ductal carcinoma. A staging PET-CT scan revealed a right supraclavicular lymph node in size of $11 \times 10 \mathrm{~mm}$ (SUVm:7.9) and there was also a nodular lesion measured $7 \times 8 \mathrm{~mm}$ on the thyroid gland. There was no axillary lymph node involvement (Figure 1).

In the absence of axillary lymph node involvement, the thyroid nodule and cervical lymph nodes made us concern about a second primary malignancy, most probably a thyroid neoplasm. Thyroid fine needle aspiration biopsy showed normal thyroid glands and tru-cut biopsy of the right supraclavicular lymph node showed granulomatous lymphadenitis which is consistent with tuberculosis lymphadenitis (Figure 2A). The PPD test measured $15 \mathrm{~mm}$ and serum quantiferon were positive. The patient was consulted to the infectious disease department and an initial intensive-phase therapy with four antibiotics (INH, RIF, PZA, EMB) and continuation-phase therapy with 2 antibiotics (INH, RFA) planned.

The patient underwent lumpectomy and axillary lymph node dissection by surgical team. The pathology of the tumor revealed, 2.8 $\mathrm{cm}$ invasive neoplasm with intact surgical margins. The axillary lymph nodes were tumor-free. The tumor showed $80 \%$ ER, PR positivity, cErbB2 was negative and Ki-67 proliferation index was \%1 (Figure 2B).

Adjuvant endocrine therapy with tamoxifene was planned for perimenopausal early stage breast cancer. However, the usage of rifampin for tuberculosis treatment concerned us about drug interactions between tamoxifene and rifampin. We started letrozole, an aromatase inhibitor and has no known drug interaction with rifampin, to our patient.

Anti-tuberculosis treatment completed six months after. Ten months after letrozole treatment we made another PET-CT scan and it showed persisting of the thyroid nodule and complete regression of cervical lymphadenopathies (Figure 1B). There were no recurrence findings on examination and imaging.

\section{Discussion}

Breast cancer is the most common cancer among women worldwide [1]. It is very unusual to detect distant lymph node metastases of breast

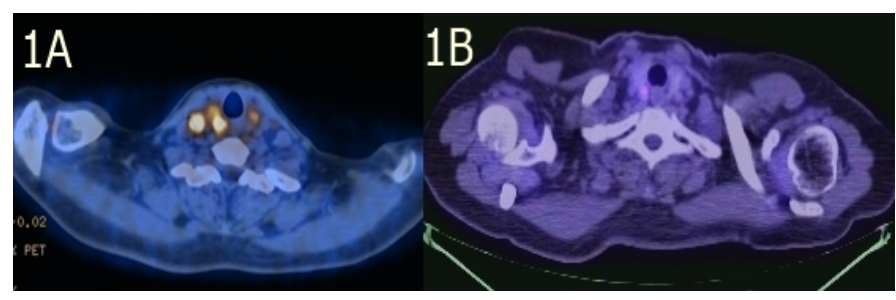

Figure 1. A is showing right cervical lymph node and thyroid involvement seen on PETCTdetected initially. B is showing the loss of FDG involvement in the right cervical lymph node in follow-up.

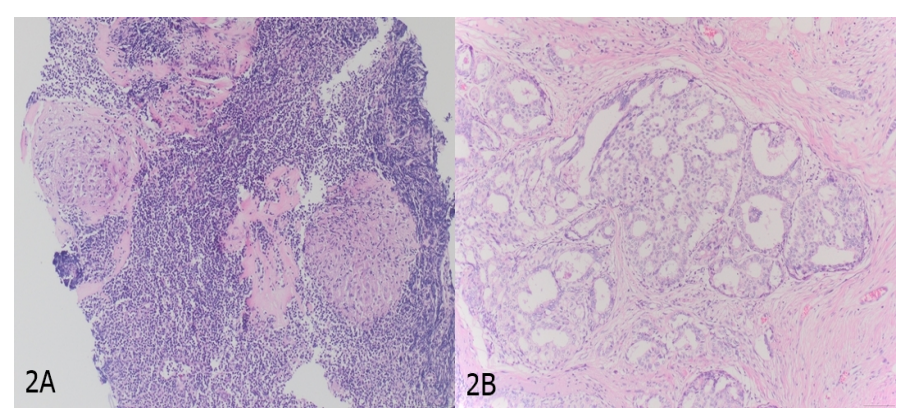

Figure 2. $\mathbf{A}$ is the fine needle aspiration biopsy of the right supraclavicular lymph node showing granulomatosis lymphadenitis $\mathbf{B}$ is the mastectomy material showing invasive ductal carcinoma of the breast.

*Correspondence to: İmdat Eroğlu M.D, Department of Internal Medicine, Hacettepe University Faculty of Medicine, Sihhiye, Ankara, 06100, Turkey, Tel: +90 54139691 84, E-mail: i.eroglu.1903@gmail.com

Received: November 13, 2019; Accepted: November 24, 2019; Published: November 27, 2019 
cancer without involvement of axillary lymph nodes [2]. In such cases, clinicians should concern about another reason of lymphadenopathy other than breast cancer metastasis. The tuberculosis might be an important cause of lymphadenitis misdiagnosed as cancer metastasis, so patient's risk factors should be considered especially in endemic areas. There would be a devastating result if we assume cervical lymph nodes as metastasis and start chemotherapy without confirmation.

Another challenge is anti-tuberculosis treatment in cancer patients due to both side effects and drug interactions. Tamoxifene, a selective estrogen receptor modulator drug, is frequently used for treatment of breast cancer. However, tamoxifen is metabolized by the CYP2D6 enzyme, so that is greatly influenced by cytochrome induction. Rifampin is a main drug in the treatment of tuberculosis and is a very potent inductor of the cytochrome p450 levels. Binkhorst et al. compared serum levels of tamoxifene and its active metabolite, endoxifene, in four patients tamoxifen administration alone and with rifampin. The results showed that rifampin administration declined serum tamoxifene leves in all patients ranging by $\% 28$ to 85 . The study strongly indicated that concurrent use of rifampin and tamoxifene should be avoided [3] .
Evaluation of treatment response among patients with tuberculosis lymphadenitis remains challenging. Active tuberculomas have increased macrophage and neutrophil activity. Therefore, 18F-FDG PET can be used in diagnosis, detection and most importantly treatment response of tuberculosis. Various studies showed the promising role of using 18F-FDG PET in the disease course [4]. In our case, the FDG involvement on the cervical region lost after six-month treatment of the tuberculosis and directed us about treatment response.

\section{References}

1. Siegel RL, Miller KD, Jemal A (2019) Cancer statistics, 2019. CA Cancer J Clin 69: 7-34. [Crossref]

2. Ullah I, Karthik G-M, Alkodsi A, Kjällquist U, Stålhammar G, et al. (2018) Evolutionary history of metastatic breast cancer reveals minimal seeding from axillary lymph nodes. J Clin Invest 128: 1355-1370. [Crossref]

3. Binkhorst L, van Gelder T, Loos WJ, de Jongh FE, Hamberg P, et al. (2012) Effects of CYP induction by rifampicin on tamoxifen exposure. Clin Pharmacol Ther 92: 62-67. [Crossref]

4. Vorster M, Sathekge MM, Bomanji J (2014) Advances in imaging of tuberculosis: the role of ${ }^{18} \mathrm{~F}-\mathrm{FDG}$ PET and PET/CT. Curr Opin Pulm Med 20: 287-293. [Crossref]

Copyright: (C2019 Eroğlu I. This is an open-access article distributed under the terms of the Creative Commons Attribution License, which permits unrestricted use, distribution, and reproduction in any medium, provided the original author and source are credited. 